\title{
Legg-Calvé-Perthes hastalığında pelvik osteotomiler
}

\section{Pelvic osteotomies in Legg-Calvé-Perthes disease}

\author{
Sancar Serbest ${ }^{1}$, Mehmet Bulut ${ }^{2}$, Lokman Karakurt ${ }^{3}$ \\ ${ }^{1}$ Kırıkkale Üniversitesi Tıp Fakültesi, Kırıkkale \\ ${ }^{2}$ Dicle Üniversitesi Tıp Fakültesi, Diyarbakır \\ ${ }^{3}$ Fırat Üniversitesi Tıp Fakültesi, Elazığ
}

\begin{abstract}
Legg-Calvé-Perthes hastalığının tüm evreleri boyunca femur başının asetabulum içerisinde tutulmasının hastalı̆̆ın seyri üzerine olumlu etkisi olduğu görüşü yaygın olarak kabul görmeye başladıktan sonra, cerrahi dışı veya cerrahi örtünme tedavileri, hastalığın tedavisinde daha çok tercih edilir hale gelmiştir. Cerrahi dışı örtünme tedavilerinin uzun sürmesi, bu amaçla kullanılan cihazların hastanın günlük aktivitelerini kısıtlaması ve yaşam kalitesini olumsuz etkilemesi nedeniyle hastalar tarafindan tolere edilebilmesi oldukça zordur. Bu nedenlerden dolayı cerrahi örtünme tedavileri seçilmiş hastalarda bir adım daha önde görünmektedir. Son zamanlarda yapılan karşılaştırmalı çalışmalar da bu görüşü destekler niteliktedir. Literatürde pelvik osteotomilerle ilgili pek çok farklı yöntem tanımlanmış olmasına rağmen, Legg-CalvéPerthes hastalığında en sık kullanılanlar; Chiari, Shelf, innominat ve üçlü pelvik osteotomilerdir. Bu osteotomiler içerisinde özellikle 6-8 yaş arasında innominat osteotomi daha fazla ön plana çıkarken, daha ileri yaşlarda varizasyon osteotomisi veya tek başına üçlü osteotomi gibi daha komplike ve kombine osteotomilerin kullanılması önerilmektedir. Ancak hangi yöntemle yapılırsa yapılsın, yaş arttıkça cerrahi tedavinin başarı oranlarında düşüş görüldüğü de inkâr edilemez bir gerçek olarak karşımızda durmaktadır.
\end{abstract}

Anahtar sözcükler: Legg-Calvé-Perthes; cerrahi tedavi; pelvik osteotomiler
Since the idea that maintaining the femoral head in the acetabulum during all stages of the Legg-Calvé-Perthes has a positive effect on the course of the disease become widely accepted, the treatment either with non-surgical or surgical containment has commonly become preferred. The long duration of non-surgical containment treatments and the devices used for this purpose are very difficult to tolerate because they restrict the daily activities of the patient and adversely affect the quality of life. For these reasons, surgical containment seems to be one step ahead in selected patients. Recent comparative studies support this view. Although many different methods of pelvic osteotomies have been described in the literature for the treatment of Legg-Calvé-Perthes, the most commonly used methods are Chiari, Shelf, innominate and triple pelvic osteotomies. Among these osteotomies, especially between 6 and 8-year-old ages the innominate osteotomy is more foreground, while in older ages, varus osteotomy or more complicated and combined osteotomies such as triple osteotomy is recommended. However, regardless of which method is preferred, it is undeniable that the success rate of surgical treatment decreases as the age increases.

Key words: Legg-Calvé-Perthes; surgical treatment; pelvic osteotomies
egg-Calvé-Perthes (LCP) hastalığında tedavinin temel amacı, femur başı küreselliğinin ve asetabular uyumun hastalıktan en az düzeyde etkilenmesini sağlamak ve genç yaşta gelişebilecek dejeneratif eklem hastalığı riskini en aza indirmektir. Genel kabul edilen görüş ise femur başının asetabulum içinde tutulmasını sağlamaktır. Bu sayede, asetabulum femur başınının şekillenmesine katkıda bulunacaktır. Perthes hastalığında en temel tedavi yöntemi, örtünme prensibinin uygulanmasıdır. Örtünmeyle, asetabulumun tamamen zayıflayan femur başını kapsaması ile femur başına etki eden kuvvetlerin eşit olarak dağılması amaçlanmaktadır. Bu ise konservatif ya da cerrahi yöntemlerle sağlanabilir. Tedavisiz takip edilen hastaların çoğunda fonksiyonel olarak iyi sonuç bildirilse de, hastalığın doğal seyri ve hastaya özgü prognostik etmenler göz önünde bulundurularak uygun müdahalenin yapılması, erişkin dönemde dejeneratif koksartroz gelişiminden korunma sağlayabilir. ${ }^{[1-6]}$

Her ne kadar kesin olarak tanımlanmamış olsa da, \%80'in üzerindeki örtünme miktarının femur başı lateral taşmayı ve kompresyonunu engellediği kabul edilir. Pelvik osteotomiye geçmeden önce, femur proksimali normal biyomekaniği tesis edilebilir. Femoral

- İletişim adresi: Dr. Mehmet Bulut, Dicle Üniversitesi Tıp Fakültesi Ortopedi ve Travmatoloji AD, Diyarbakır Tel: 0505 - 9886675 e-posta: bulmeh@yahoo.com

- Gelis tarihi: 20 Subat 2017 Kabul tarihi: 20 Subat 2017 
anteversiyon veya retroversiyon rotasyonel osteotomi ile, boyun diyafiz açısı farklılıkları ise varus-valgus osteotomileri ile düzeltilebilir. Proksimal femurun normal anatomisi ve biyomekaniği sağlandıktan sonra, asetabular displazi varsa, femur başının örtünmesini arttırmak amacıyla pelvik osteotomiler düşünülmelidir. ${ }^{[7-9]}$

Cerrahi örtünme tedavisi uygulanması için, kalça hareketlerinin açık olması ve menteşeli abduksiyonun olmaması ise ön şartlardandır. Pelvik osteotomilerden sonra; tespit kaybı, distal fragmanın kayması, bacağın uzaması, kalça fleksiyonunun azalması, eklem sertliği, menteşeli abduksiyon ve aşırı örtünmeye bağlı sıkışma sendromu gibi komplikasyonların görülebileceği bildirilmiştir. ${ }^{[1,10]}$

Suleima ve arkadaşlar. ${ }^{[11]}$ çocukluk çağında pelvik osteotomi geçiren hastalarda asetabular aşırı örtünmenin sık görüldügünü, ancak retroversiyon ve kalça ağrısı arasında bir korelasyon olmadığını bildirmişlerdir.

LCP hastalığının ilk tanımlanmasının üzerinden 100 yıldan daha fazla bir zaman geçmiş olmasına rağmen, hastalıkla ilgili pek çok konu tam olarak aydınlatılamamış ve yazarlar arasında fikir birliği sağlanamamıştır. Üzerinde büyük çoğunlukla ortak kanaat oluşan konulardan birisi, örtünme tedavisinin hastalığın seyrini ve sonuçlarını olumlu yönde etkilediğidir. ${ }^{[1,5,7,8,12-19]}$

Cerrahi örtünme tedavisi, 1960 yılında femoral varizasyon osteotomisiyle başlamış olup günümüze kadar süregelmiştir. Salter daha sonra, innominat osteotomi uygulamasıyla örtünme prensibine farklı bir bakış açısı kazandırmıştır. İleri derecede tutulumu olan Perthes hastalarında örtünme prensibinin sağlanması için, femoral ve pelvik osteotomilerin kombine kullanımı da gündeme gelmiştir. ${ }^{[20-23]}$

Salter ${ }^{[16]} 1966$ yılında yaptığı deneysel çalışma sonrasında, biyolojik plastisite kavramını tanımlamıştır. Bu teoriye göre; hastalık başladıktan sonraki süreçte, femur başı asetabulum içerisinde tutulursa normal veya normale daha yakın bir baş elde edilebilir. Bu teoriden yola çıkarak, cerrahi ve cerrahi dışı örtünme tedavileri geliştirilmiştir. Biz bu bölümde, pelvik osteotomilerle yapılan cerrahi örtünme tedavilerinden bahsedeceğiz.

\section{PELVIK OSTEOTOMI YÖNTEMLERI}

1. Yüzey arttırıcı ameliyatlar: (Salvage yöntemler, mediyal deplasman osteotomileri)
a. Chiari osteotomisi
b. Kawamura osteotomisi
c. Asetabular çatı (shelf) girişimleri:
i. Staheli
ii. Wilson

2. Yeniden yönlendirici osteotomiler (komplet)

a. Tekli pelvik osteotomi (Salter) b. İkili pelvik osteotomi

(Sutherland ve Greenfield, Hopf)

c. Üçlü pelvik osteotomi

(Steel, Tönnis, Tachdjian, LeCoeur, Carlioz)

d. Poligonal üçlü pelvik osteotomi (Kotz)

e. Periasetabular osteotomi (Ganz, )

f. Sferik osteotomiler (Nishio, Wagner, Eppright)

g. Rotasyonel asetabular osteotomi (RAO)

3. Yeniden şekil verici osteotomiler (inkomplet)

a. Pemberton osteotomisi (perikapsüler osteotomi)

b. Dega osteotomisi

Literatürde pelvik osteotomilerle ilgili pek çok farklı yöntem tanımlanmış olmasına rağmen, LCP hastalığında en sık kullanılanlar olan, Chiari, shelf, innominat ve üçlü pelvik osteotomiden bahsedeceğiz.

\section{Chiari Osteotomisi}

Chiari ameliyatı, iyileşmekte olan ancak lateralize kalmış femur başını örtmek amacıyla kullanılır. LCP hastalığında Chiari osteotomisi, bazı yazarlar tarafından primer ameliyat olarak tanımlanmıştır. Özellikle femur başında ciddi deformitesi olan, asetabulum ve femur arasında uyumsuzluğun olduğu daha büyük çocuklarda, ağrılı kalçaların tedavisinde önerilmektedir. [24-27] Bununla birlikte, cerrahi teknikteki zorluklar, yöntemin güvenilir olmaması ve gerçek yararının bilinmemesinden dolayı sık kullanılan bir teknik değildir. Çünkü, hastalar ameliyat edilmediğinde dahi iskelet matürasyona yaklaştıkça femur başının yuvarlaklığı sıklıkla spontan olarak düzelmektedir. ${ }^{[28,29]}$

\section{"Shelf" Asetabuloplasti}

LCP hastalığında shelf asetabuloplastisi 1940 yılından bu yana kullanılmaktadır. Farklı tekniklerle shelf asetabuloplastisi, örtünme prensibini sağlamak amacı ile uygulanmaktadır. Shelf asetabuloplastisi hastalığın erken dönemlerinde birincil örtüme tedavisi olarak kullanılmakla beraber, femur başının lateral subluksasyonu, yetersiz örtünme ve menteşeli abduksiyon gibi endikasyonlarda da uygulanmaktadır. ${ }^{[13,30,31]}$

Shelf asetabuloplasti tekniğinin diğer cerrahi yöntemlere göre avantajları; ileri düzeyde örtünme sağlayabilmesi ve bu sayede lateral subluksasyonun önlenebilmesi, femoral osteotomiyle karşılaştırıldığında kısalık oluşturmaması ve abduktor kurvet kolunu bozmaması, pelvik osteotomilerle karşılaştırıldığında daha az cerrahi deneyim gerektirmesi ve daha az komplikasyon oranlarına sahip olması olarak sıralanabilir. ${ }^{[32-34]}$ Genellikle, diğer cerrahi tedavilerin başarısızlığı durumunda uygulanır. ${ }^{[30]}$ Bazı cerrahlar, örtünme için ilk yapılacak cerrahinin shelf asetabuloplasti olması gerektiğini belirtir ve hastalığın başlamasından sonraki altı aylık dönem içerisinde yapılmasını önerirler. ${ }^{[31,35]}$ 
Gill[36], ameliyatı asetabulumun üzerine derin bir çentik açıp tavanı buradan aşağıya doğru kanırtarak yapmayı tarif etmiştir. Staheli ise slot tekniğini geliştirmiştir. Ameliyatın komplikasyonları olarak; geniş greft konulmasına bağlı olarak abduksiyon kısıtılığı, ince greft konulmasına bağlı olarak ise yetersiz örtünme sayılabilir. ${ }^{[37]}$

Shelf asetabuloplastisinin femur başının örtünmesinde düzelmeye neden olduğu gösterilmiştir. Ancak literatürde, bu prosedürün osteoartriti engellediği ve uzun dönemde fonksiyonel sonuçları düzelttiğiyle ilgili yeterli bilgi bulunmamaktadır. Son zamanlarda, Shelf asetabuloplastisinin bir türü olan labral destek tekniği, örtünmeyi sürdürmek amacıyla önerilmektedir. Minimal insizyonla yapılan labral destek, shelf asetabuloplastisinin bir varyasyonu olup artroskobik görüş altında bir raf üzerine allogreftle destek etkisi oluşturulmasından ibarettir. Bu minimal insizyon tekniğinin sonuçları klasik yöntemle karşılaştırılmış ve her iki yöntemin sonuçlarının da Petrie alçısı, femoral varus osteotomisi ve Salter'in innominat osteotomisiyle benzer olduğu görülmüştür. Labral destek prosedürünün diğer avantajı, cerrahi sonrası gerçek asetabulumun ilave lateral büyümesiyle daha fazla örtünme oluşturmasıdır. Böylece, bu teknikle subluksasyon önlenebilir ve lateral asetabular büyüme uyarılabilir. ${ }^{[38-40]}$

Carsi ve arkadaşları ${ }^{[41]}$, shelf asetabuloplasti ile tedavi ettikleri 45 olguluk çalışmalarında; cerrahi tedavi endikasyonunda deformitenin derecesinin ve Rimer migrasyon indeksinin göz önünde bulundurulmasının önemine ve erken evrede sonuçların iyi olduğuna vurgu yapmışlardır.

Kadhim ve arkadaşları ${ }^{[42]}$, LCP hastalarında erken (Waldenstrom I ve II, 204 kalça) ve geç (Waldenstrom III ve IV, 62 kalça) evrede shelf asetabuloplastisi yapılan hastaların sonuçlarını değerlendirdikleri meta-analiz bir çalışmada, erken evrede ameliyat edilenlerde \%85 oranında iyi sonuç (Stulberg Tip I, II, III), geç evrede ameliyat edilenlerde ise $\% 69$ oranında iyi sonuç bildirmişler ve 10-11 yaş üzerinde shelf prosedürü uygulandığında ihtiyatlı olmayı önermişlerdir.

\section{İnnominat Osteotomi}

LCP hastalığında innominat osteotomi, ilk kez 1962 yılında Salter tarafından uygulanmıştır. ${ }^{[43,44]}$ Günümüzde de, tüm dünyada yaygın olarak kullanılmaktadır. Salter, asetabular kötü yönelimin femur başının örtünme yetersizliğinden sorumlu olduğunu ileri sürmüş ve asetabuluma yeniden yön vererek, fonksiyonel yük verir pozisyonda stabil bir kalça elde etmek için bu osteotomiyi tasarlamıştır. Salter osteotomisinde asetabular fragmanın mobilitesi, esnek dayanak noktası olarak symphisis pubis'ten rotasyon yaptırılmasına bağlıdır. ${ }^{[15,16]}$

Orijinal olarak tanımlanan tekniğe ek olarak, kapsül açılmaması ve iliopsoasın uzatılması önerilmiştir. Adduktor kaslar gergin ise uzatılmalıdır. Bazı merkezlerde, Salter ameliyatının Kalamchi modifikasyonu tercih edilir. Bu teknikte, kesilen distal parça iliak kemikte oluşturulan oluğa yerleştirilir. Bu yöntemin avantajı, pelvisi uzatmadan femur başının aşırı basınca maruz kalmasını önleyerek asetabuluma pozisyon vermesidir. Iliak osteotominin komplikasyonları ise; fiksasyon kaybı sonucu distal parçanın deplase olması, bacağın uzaması, kalça fleksiyonunun azalması ve eklem sertliğinin gelişebilmesidir. Ameliyat sonrasında menteşeli abduksiyon gelişebilir. ${ }^{[13,43-45]}$

Son yıllarda, bu yöntemle yapılan tedavilerde başarılı sonuçlar bildirilmiştir. İyi sonuçların genellikle 6-8 yaş arasında Lateral Pillar sınıflamasına göre Tip $B$ ve $B / C$ kalçalarla ilgili olduğu, Tip $C$ tipi kalçalarda ise sonuçların daha kötü olduğu vurgulanmıştır. ${ }^{[14,46]}$

Herring ve arkadaşları ${ }^{[47]}$, farklı tedavi yöntemlerini karşılaştırdıkları çok merkezli ileriye dönük çalışmada, başlangıç yaşları 6-8 yıl arasında olan olgularda en başarılı sonuçları Salter osteotomisiyle tedavi ettikleri grupta bulmuşlardır (\%69 Stulberg Tip I ve II).

Yavuz ve arkadaşları ${ }^{[48]}$, yaş ortalaması 8,8 ve ameliyat zamanında Herring'e göre yedisi Tip B, üçü Tip $B / C$ ve sekizi Tip C olan 18 hastayı Salter innominat osteotomi yöntemiyle tedavi etmişlerdir. Ortalama 78 aylık takip sonrasında, 10 olgu Stulberg I ve II, yedi olgu Stulberg III ve bir olgu Stulberg IV olarak değerlendirilmiş ve Salter osteotomisinin geç başlangıçlı LCP hastalarında başarılı bir yöntem olduğunu bildirmişlerdir.

Kliniğimizde; başlangıç yaşı 6-10 arasında, Herring'e göre Tip $B, B / C$, ve $C$, olup ameliyat sırasında yaş ortalaması 8,1 olan 16 hasta (15 erkek, $1 \mathrm{kIz}$ ), Salter'in innominat osteotomisi uygulayarak tedavi edildi. Ortalama takip süresi 51 ay olan çalışmada, Stulberg sınıflamasına göre son radyografik değerlendirmede, dokuz kalçada $(\% 56,25)$ iyi (Stulberg $\mathrm{I} / \mathrm{II})$, beş kalçada $(\% 31,25)$ orta (Stulberg III) ve iki kalçada $(\% 12,5)$ kötü (Stulberg IV) sonuç mevcuttu (Şekil 1). Herring lateral kolon sınıflamasına göre $B$ veya $B / C$ olan 12 olgunun sekizinde $(\% 66,6)$ iyi sonuç (Stulberg I/II), C olarak sınıflandırılan dört olgunun da sadece birinde (\%25) iyi bir sonuç mevcuttu. İyi sonuçlar, Herring'e göre $B$ ve $B / C$ olan kalçalar ve sekiz yaş altındaki olgularda alındı. Tip $C$ ve sekiz yaş üstündeki olgularda sonuçlar daha kötü idi ve bu olgularda tek başına Salter pelvik osteotominin yetersiz olduğu kanaatine varıldı (Şekil 1 ve 2). ${ }^{[14]}$ 

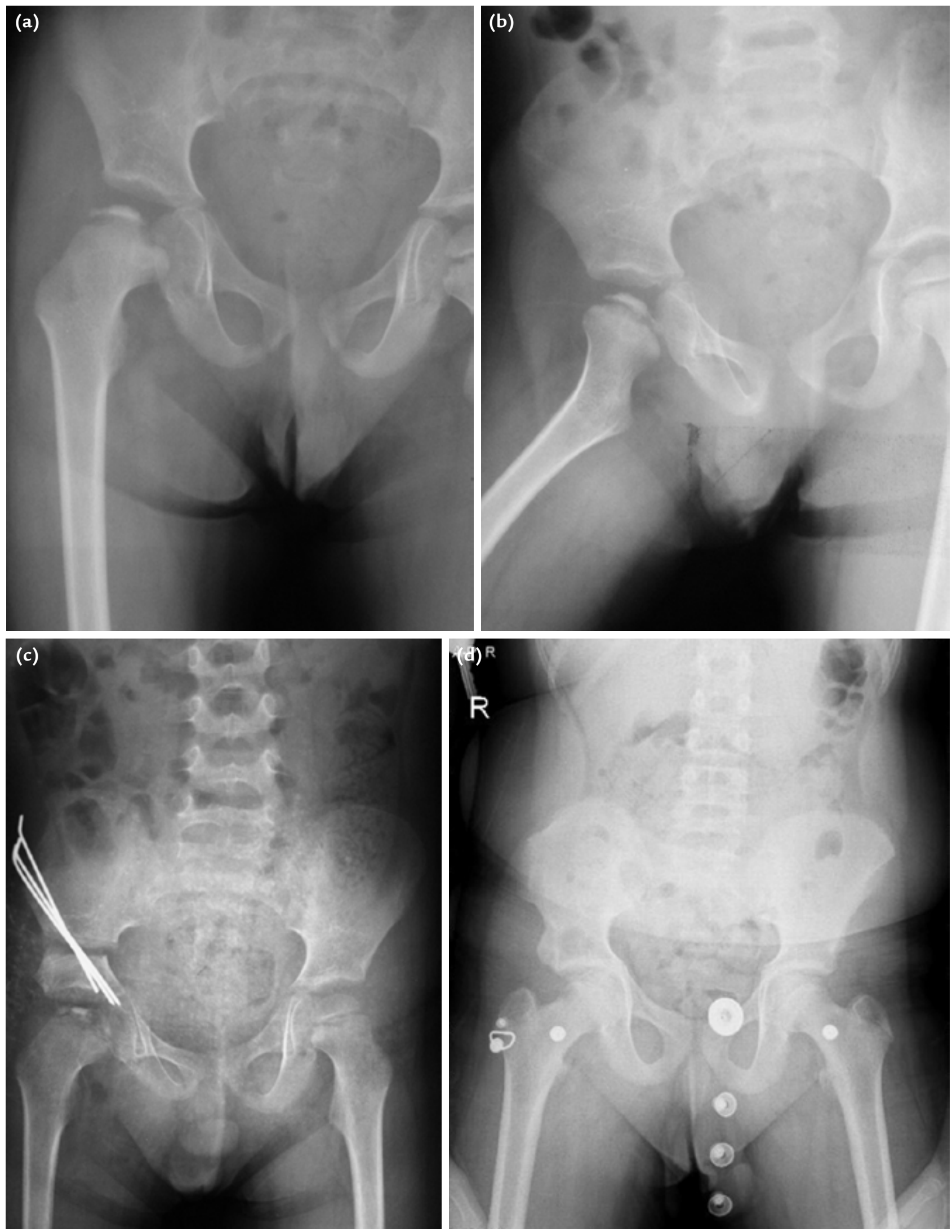

Şekil 1. a-d. Beş yaş erkek, sağ Herring tip C, ameliyat öncesi grafisi (a, b). Sağ Salter osteotomisi + iliopsoas tenotomisi sonrası erken dönem radyografisi (c). Ameliyat sonrası 10. yıldaki grafisi (d), Stulberg Tip I. 

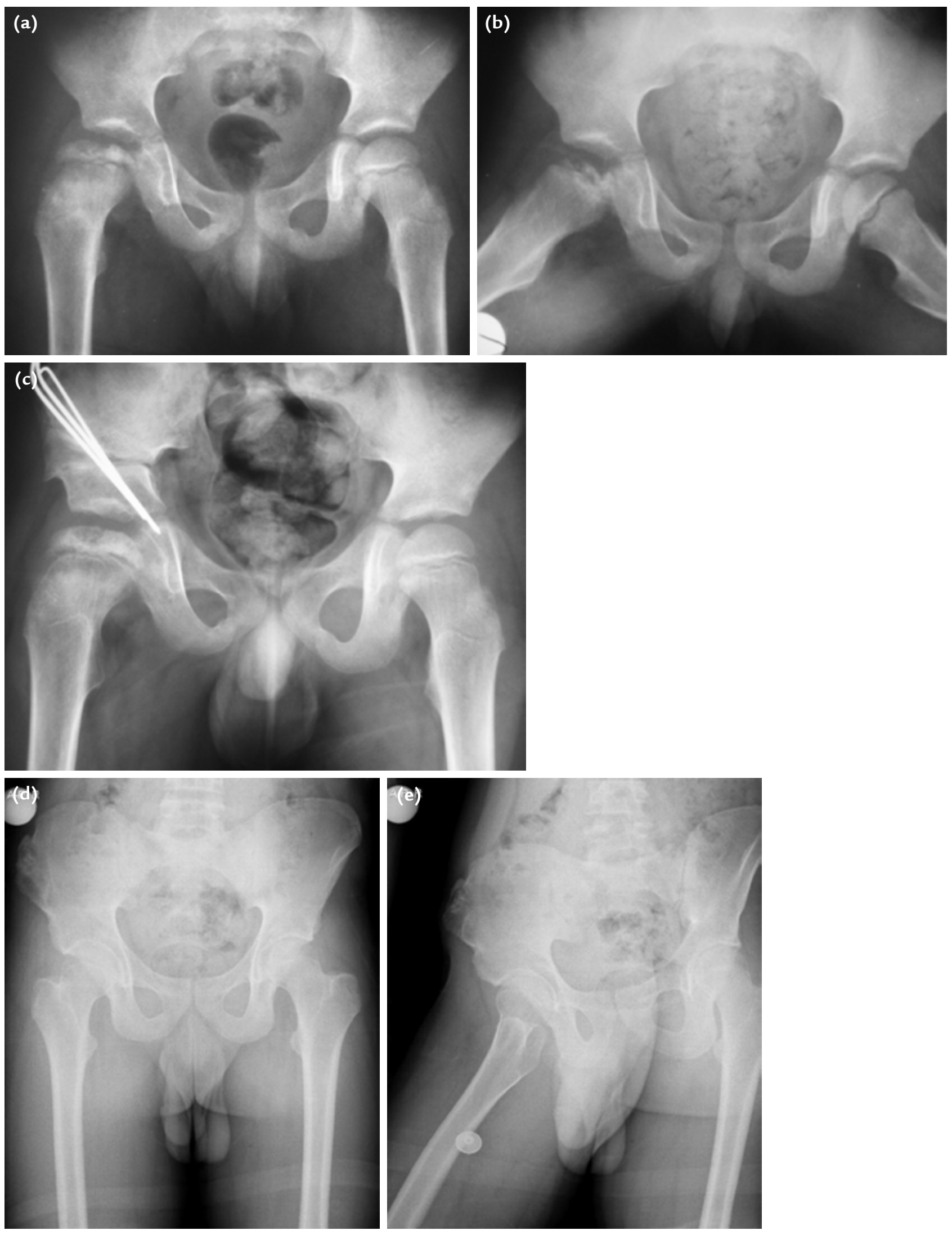

Şekil 2. a-e. Yedi yaşında erkek, Herring tip B kalçanın ameliyat öncesi radyografisi (a, b). Salter osteotomisi + iliopsoas tenatomisi sonrası erken dönem radyografisi (c). Ameliyat sonrası 10. yıldaki ön-arka (d) ve yan (e) radyografileri, Stulberg Tip II. 

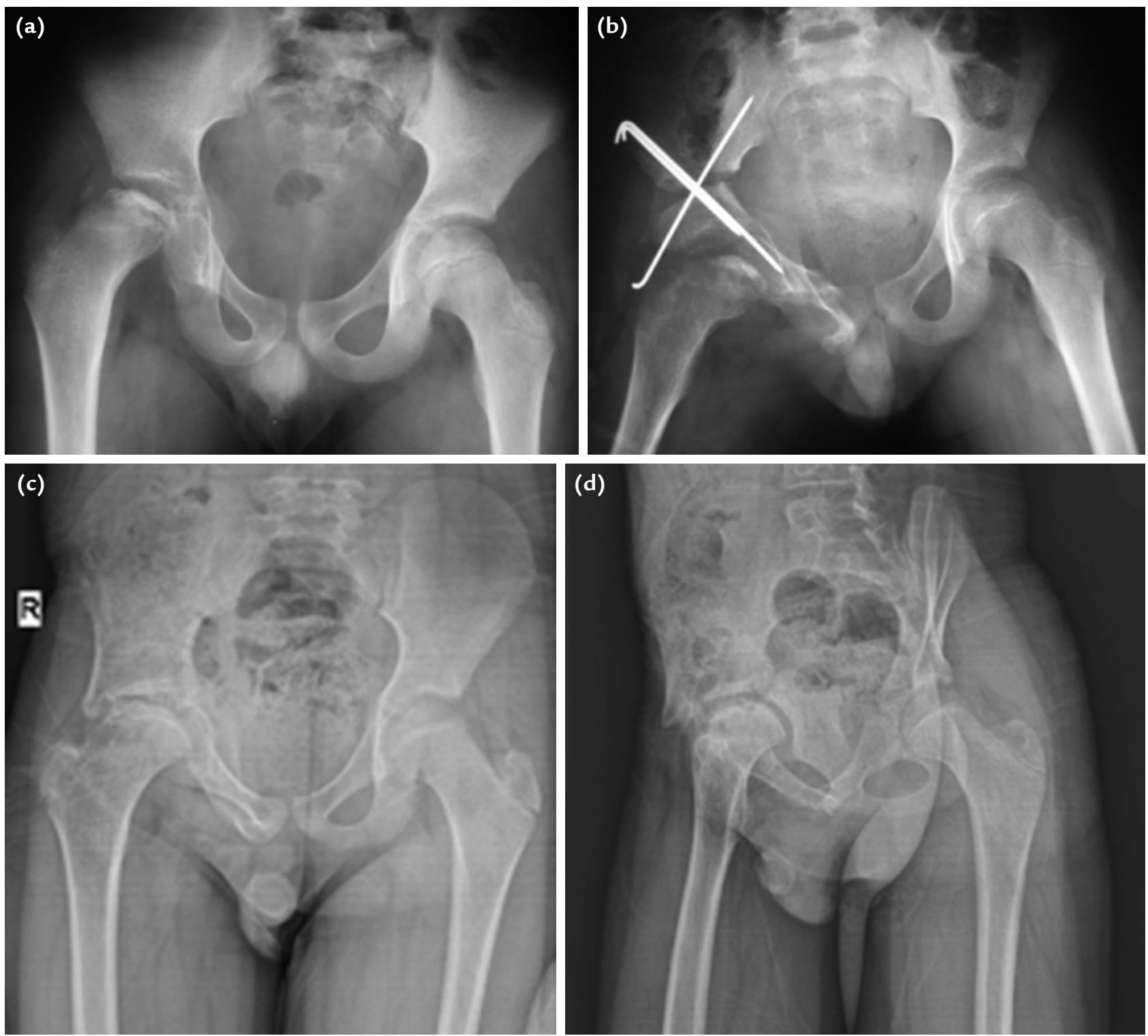

Şekil 3. a-d. Sekiz yaşında erkek hasta Herring Tip C (a). Üçlü osteotomy + iliopsoas + adduktor tenotomi sonrası erken radyografisi (b). Dördüncü yıldaki ön-arka (c) ve yan (d) grafisi, Stulberg Tip III.

\section{Üçlü Pelvik Osteotomi}

Tekli pelvik osteotomi, asetabular displazide özellikle symphisis pubis kapandıktan sonraki yaşlarda esnekliğin azalmasına bağlı olarak asetabular örtünmede yetersiz kaldığı için, Le Coueur, Hopf ve Steel birbirinden bağımsız olarak, üç kesimli pelvik osteotomiyi tanımlamışlardır. Steel, 1965 yılında, Salter osteotomisine ek olarak iskium ve pubis osteotomisini eklemiştir. ${ }^{[49,50]}$ 1981 yılında Tönnis, Steel'in üçlü pelvik osteotomisini modifiye etmiştir. Tekli osteotomilerde olduğu gibi, konsantrik redüksiyon bu osteotomi için de ön şarttır. Eğer iskiumdaki osteotomi, lateralden mediyale oblik olacak şekilde yapılırsa, asetabular fragman daha mediyalize edilerek, kalça eklem merkezi daha fizyolojik pozisyona getirilir ve yürüme mekanizması daha iyi düzeltilebilir. ${ }^{[17,51]}$

Steel'in yaptığı üçlü osteotomide, iskium kesisi asetabulumdan uzakta kalır (Şekil 3). Tönnis'in yönteminde ise kesi asetabuluma daha yakındır. Tönnis, iskium osteotomisini oblik yapıp ayn yerden kemik eksizyonu yaparak, daha çok rotasyon, dolaysıyla daha fazla düzelme elde etmiştir. Steel'in orijinal tekniğinde, iskium osteotomize edilirken rezeksiyon yapılmadığından, asetabulumun lateralizasyon problemi olabilmektedir. ${ }^{[52]}$ 
Steel'in tekniğini modifiye ederek iskiumdan rezeksiyon yapanlar, bu problemin bu şekilde ortadan kalktığını bildirmişlerdir. Tönnis'in üçlü osteotomi tekniğinde yapılan farklı modifikasyonlar bildirilmiştir. İskium, posterior yaklaşımla ve sakrotuberal bağların üzerinden oblik olarak kesilir, pubis osteotomisi ayrı bir transvers insizyonla yapılır. iliak osteotomi, Salter osteotomisi gibi yapılmaktadır. İskium osteotomisi perianal bölgeden uzakta yapılan bir insizyon ile yapıldığından, kontaminasyon riski yoktur. İskium ve pubis kolu osteotomileri asetabuluma yakın olarak yapıldığından, asetabulumun serbestleşme ihtimali fazladır. Yine, iskium osteotomisi oblik olarak yapıldığından, yüzey artar ve psödoartroz riski azalır. ${ }^{[52,53]}$

Stepanoviç ve arkadaşları ${ }^{[54]}$, üçlü osteotomi ile tedavi ettikleri 56 kalçanın sonuçlarını yayımlamışlardır. Ameliyat öncesi kalçaların \%70'i B veya B/C, \%30'u Tip C; son kontrollerde ise kalçaların \%64'ü Stulberg I ve ya II, \%25'i Stulberg III ve \%11'i Stulberg IV veya V olarak değerlendirilmiştir. Yazarlar, Herring sınıflamasına göre Lateral Pillar B ve B/C olanların ve sekiz yaş altında olanların sonuçlarının, Lateral Pillar $C$ ve sekiz yaş üzerinde olanlara göre daha iyi olduğunu bildirmişlerdir.

Pailhe ve arkadaşları ${ }^{[5]}$, üçlü pelvik osteotomiyle tedavi ve ortalama 15 yıl takip ettikleri 45 LCP hastasında, eklem uyumu ve başın örtünme oranını \%85 olarak bildirmişlerdir. Uzun dönem kötü sonuçların, tanı anındaki yaşla ilgili olduğunu vurgulamışlardır.

Üçlü pelvik osteotomi, LCP hastalığında örtünmenin sağlanmasında diğer bir seçenektir. Tüm olgularda femur başının örtünmesinde en etkili yöntemdir. Bununla beraber, aşırı örtünme Pincer tipi sıkışma sendromuna yol açabileceğinden, merkez-kenar açısı $44^{\circ}$ 'den daha fazla olacak şekilde düzeltme önerilmemektedir. ${ }^{[51]}$

\section{SONUÇ}

Pelvik osteotomilerin örtünme amacıyla kullanılaca$\breve{g}_{\text {I }}$ ideal hasta tanımı ve standart tedavi metodlarından bahsetmek olanaklı değildir. Ancak, hastalarda tedavi öncesinde gerekli şartların sağlanması önemlidir. Hangi cerrahi teknik uygulanırsa uygulansın, ameliyat öncesi asetabulum ve femur başı uyumunun olması, maksimum eklem hareket açıklığının sağlanması, abduksiyonda femur başının örtünmesi, ön şartlardandır. Pelvik osteotominin aynı taraf alt ekstremitede uzunluk artışına yol açtığı durumlarda rutin olarak iliopsoas uzatması ve adduktor kontraktür varlığında ise adduktor tenotomi eklenmesi de vurgulanması gereken diğer önemli konulardır.

Pelvik osteotomilerle yapılan çalışmaların çoğunun ortak noktası; başlangıç yaşının ileri olduğu ve Herring lateral kolon sınıflamasına göre Tip C olan olguların sonuçlarının kötü olduğu yönündedir.

Son yıllarda perfüzyon manyetik rezonans (MR) görüntüleme ile yapılan çalışmalar, hastalığın prognozunu tahmin etmede ve hangi olgulara cerrahi uygulamanın gerekeceğini öngörme anlamında ümit verici olarak görülmektedir.[56,57]

Mevcut bilgiler ışığında; 6-9 yaş aralığı başlangıcında, Herring sınıflamasına göre Tip $B$ ve $B / C$ ve kötü prognoz riski mevcut olan (lateral taşma, perfüzyon MR görüntülerde iskemik alanın fazla olması vb.) olgularda pelvik osteotomiler, prognoz üzerine olumlu etki eden tedavi yöntemleri olarak görünmektedir. Ancak, etiyolojinin tam olarak aydınlatılmasının ve buna yönelik tedavi seçeneklerinin geliştirilmesinin, LCP'nin tedavisinde daha başarılı sonuçları beraberinde getireceği unutulmamalıdır.

\section{KAYNAKLAR}

1. Yıldırım T, Akpınar E. Legg-Calvé-Perthes hastalığı. Totbid Dergisi 2014;13:431-40. Crossref

2. Brotherton BJ, McKibbin B. Perthes' disease treated by prolonged recumbency and femoral head containment: a long-term appraisal. J Bone Joint Surg Br 1977;59(1):8-14.

3. Kamegaya M. Nonsurgical treatment of Legg-Calvé-Perthes disease. J Pediatr Orthop 2011;31(2 Suppl):S174-7. Crossref

4. Aksoy MC, Caglar O, Yazici M, Alpaslan AM. Comparison between braced and non-braced Legg-Calvé-Perthes-disease patients: a radiological outcome study. J Pediatr Orthop B 2004;13(3):153-7.

5. Westhoff B, Martiny F, Krauspe R. Perthes Disease. Z Orthop Unfall 2014;152(6):617-33. Crossref

6. Joseph B. Management of Perthes' disease. Indian J Orthop 2015;49(1):10-6. Crossref

7. Wainwright AM, Catterall A. Legg-Calvé-Perthes Disease. In: Benson M, Fixsen J, Macnicol M, Parsch K, editors. Children's Orthopaedics and Fractures, 3rd ed. New York, NY: Springer; 2010. p.465-80.

8. Weinstein SL, Flynn JM. Lovell WW, editor. Lovell \& Winter's Pediatric Orthopaedics, 7th ed. Philadelphia: Wolters Kluwer Health / Lippincott Williams \& Wilkins; 2013.

9. Herring J, editor. Tachdjian's Pediatric Orthopaedics: From the Texas Scottish Rite Hospital for Children. Saunders; 2013.

10. Coleman S, Kehl D. An evaluation of Perthes Disease: comparison of non-surgical and surgical means. Presented at the 48th Annual meeting of the American Academy of Orthopaedics Surgeons. Las Vegas, February 26 to March 3, 1981.

11. Al-Moussa S, Moroz P, Beaulé PE. Effect of pelvic osteotomy in the skeletally immature on acetabular coverage. HSSJ 2012;8(3):235-9. Crossref

12. Mazloumi SM, Ebrahimzadeh MH, Kachooei AR. Evolution in diagnosis and treatment of Legg-Calvé-Perthes disease. Arch Bone Jt Surg 2014;2(2):86-92. 
13. Chaudhry S, Phillips D, Feldman D. Legg-Calvé-Perthes disease: an overview with recent literature. Bull Hosp Jt Dis (2013) 2014;72(1):18-27.

14. Bulut M, Demirtş A, Uçar BY, Azboy I, Alemdar C, Karakurt L. Salter pelvic osteotomy in the treatment of Legg-CalvéPerthes disease: the medium-term results. Acta Orthop Belg 2014;80(1):56-62.

15. Salter RB. Innominate ostetotomy in the treatment of congenital hip dislocation and subluxation of the hip. J Bone Joint Surg Br 1961;43:518-39.

16. Salter RB. Role of innominate osteotomy in the treatment of congenital dislocation and subluxation of the hip in older child. J Bone Joint Surg Am 1966;48(7):1413-39.

17. Price CT, Thompson GH, Wenger DR. Containment methods for treatment of Legg-Calvé-Perthes disease. Orthop Clin North Am 2011;42(3):329-40. Crossref

18. Aksoy MC. Legg Calvé Perthes Hastalığının Tedavisinde, Yöntem Seçimi ve Zamanlaması. Turkiye Klinikleri J Orthop \& Traumatol-Special Topics 2010;3(4):47-52.

19. Herring JA. Legg-Calvé-Perthes disease at 100: a review of evidence-based treatment. J Pediatr Orthop 2011;31(2 Suppl):S137-40. Crossref

20. Axer A. Subtrochanteric osteotomy in the treatment of Perthes' disease: a preliminary report. J Bone Joint Surg Br 1965;47:489-99.

21. Atsumi T, Yoshiwara S. Rotational open wedge osteotomy in a patient aged older than 7 years with Perthes' disease -a preliminary report. Arch Orthop Trauma Surg 2002;122(6):346-9.

22. Wenger DR, Pandya NK. A brief history of Legg-Calvé- Perthes disease. J Pediatr Orthop 2011;31(2 Suppl):S130-6. Crossref

23. Bhuyan BK. Early outcomes of one-stage combined osteotomy in Legg-Calvé-Perthes disease. Indian J Orthop 2016;50(2):183-94. Crossref

24. Cahuzac JP, Onimus M, Trottmann F, Clement JL, Laurain JM, Lebarbier P. Chiari pelvic osteotomy in Perthes disease. J Pediatr Orthop 1990;10(2):163-6.

25. Chiari K. Medial displacement osteotomy of the pelvis. Clin Orthop Relat Res 1974;(98):55-71.

26. Lack W, Feldner-Busztin H, Ritschl P, Ramach W. The results of surgical treatment for Perthes' disease. J Pediatr Orthop 1989;9(2):197-204.

27. Bailey TE Jr, Hall JE. Chiari medial displacement osteotomy. J Pediatr Orthop 1985;5(6):635-41.

28. Bennett JT, Mazurek RT, Cash JD. Chiari's osteotomy in the treatment of Perthes' disease. J Bone Joint Surg $\mathrm{Br}$ 1991;73(2):225-8.

29. Herring JA, Williams JJ, Neustadt JN, Early JS. Evolution of femoral head deformity during the healing phase of LeggCalvé-Perthes disease. J Pediatr Orthop 1993;13(1):41-5.

30. Kruse RW, Guille JT, Bowen JR. Shelf arthroplasty in patients who have Legg-Calvé-Perthes disease. A study of long-term results. J Bone Joint Surg Am 1991;73(9):1338-47.

31. Willet K, Hudson I, Catterall A. Lateral shelf acetabuloplasty: an operation for older children with Perthes' disease. J Pediatr Orthop 1992;12(5):563-8.

32. Freeman RT, Wainwright AM, Theologis TN, Benson MK. The outcome of patients with hinge abduction in severe Perthes disease treated by shelf acetabuloplasty. J Pediatr Orthop 2008;28(6):619-25. Crossref
33. Rab GT. Theoretical study of subluxation in early Legg-CalvéPerthes disease. J Pediatr Orthop 25(6):728-33.

34. Pecquery R, Laville JM, Salmeron F. Legg-Calvé-Perthes disease treatment by augmentation acetabuloplasty. Orthop Traumatol Surg Res 2010;96(2):166-74. Crossref

35. Lim KS, ShimJS. Outcomes of Combined ShelfAcetabuloplasty with Femoral Varus Osteotomy in Severe Legg-Calvé-Perthes (LCP) Disease: Advanced Containment Method for Severe LCP Disease. Clin Orthop Surg 2015;7(4):497-504. Crossref

36. Gill AB. Plastic construction of an acetabulum congenital dislocation of the hip the shelf operation. J Bone Joint Surg 1935; 17:48-59.

37. Staheli LT, Chew DE. Slotted acetabular augmentation in childhood and adolescence. J Pediatr Orthop 1992;12(5):569-80.

38. Hsu JE, Baldwin KD, Tannast M, Hosalkar $H$. What is the evidence supporting the preven-tion of osteoarthritis and improved femoral coverage after shelf procedure for Legg-Calvé-Perthes disease? Clin Orthop Relat Res 2012;470(9):2421-30. Crossref

39. Bowen JR, Guille JT, Jeong C, Worananarat P, Oh CW, Rodriquez A, Holmes L, Rogers KJ. Labral support shelf arthroplasty for containment in early stages of Legg-CalvéPerthes disease. J Pediatr Orthop 2011;31(2 Suppl):S206-11. Crossref

40. Domzalski ME, Glutting J, Bowen JR, Littleton AG. Lateral acetabular growth stimulation following a labral support procedure in Legg-Calvé-Perthes disease. J Bone Joint Surg Am 2006;88(7):1458-66.

41. Carsi B, Judd J, Clarke NM. Shelf acetabuloplasty for containment in the early stages of Legg-Calvé-Perthes disease. J Pediatr Orthop 2015;35(2):151-6. Crossref

42. Kadhim M, Holmes L Jr, Bowen JR. The role of shelf acetabuloplasty in early and late stages of Perthes disease: a meta-analysis of observational studies. J Child Orthop 2012;6(5):379-90. Crossref

43. Salter RB. The present status of surgical treatment for LeggPerthes disease. J Bone Joint Surg 1984;66(6):961-6.

44. Salter RB, Hansson G, Thompson GH. Innominate ostetotomy in the manegement of residual congenital subluxasyon of the hip in young adults. Clin Orthop Relat Res 1984;(182):53-68.

45. Kalamchi A. Modified Salter osteotomy. J Bone Joint Surg 1982;64(2):183-7.

46. Toma HF, de Almeida Oliveira Felippe Viana T, Meireles RM, Borelli IM, Blumetti FC, Takimoto ES, Dobashi ET. Comparison of the results from simple radiography, from before to after Salter osteotomy, in patients with LeggCalvé-Perthes disease. Rev Bras Ortop 2014;49(5):488-93. Crossref

47. Herring JA, Kim HT, Browne R. Legg-Calvé-Perthes disease. Part II. prospective multicenter study of the effect of treatment on outcome. J Bone Joint Surg Am 2004;86-A(10):2121-34.

48. Yavuz U, Demir B, Yildirim T, Beng K, Karakas ES Salter innominate osteotomy in the treatment of late presentation Perthes disease. Hip Int 2014;24(1):39-43. Crossref

49. Steel $\mathrm{HH}$. Triple osteotomy of the innominate bone. J Bone Joint Surg Am 1973;55(2):343-50.

50. Steel $\mathrm{HH}$. Triple osteotomy of the innominate bone: a procedure to accomplish coverage of the dislocated or subluxated femoral head in the older patient. Clin Orthop Relat Res 1977;(122):116-27. 
51. Hosalkar H, Munhoz da Cunha AL, Baldwin K, Ziebarth K, Wenger DR. Triple innominate osteotomy for Legg-CalvéPerthes disease in children: does the lateral coverage change with time? Clin Orthop Relat Res 2012;470(9):2402-10. Crossref

52. Tönnis D, Behrens K, Tscharani F. A new technique of triple osteotomy for turning displastic asetabula in adolescents and adults. Z Orthop Ihre Grenzgeb 1981;119(3):253-65.

53. Faciszewski T, Coleman SS, Biddulph G. Triple innominate osteotomy for acetabular dysplasia. J Pediatr Orthop 1993;13(4):426-30.

54. Stepanovich M, Upasani W, Bomar JD, Wenger DR. Advanced Containment With Triple Innominate Osteotomy in Legg-Calvé-Perthes Disease: A Viable Option Even in Severe Cases. J Pediatr Orthop 2015. [Epub ahead of print]
55. Pailhe R, Cavaignac E, Murgier J, Cahuzac JP, de Gauzy JS, Accadbled F. Triple osteotomy of the pelvis for Legg-CalvéPerthes disease: a mean fifteen year follow-up. Int Orthop 2016;40(1):115-22. Crossref

56. Sankar WN, Thomas $S$, Castañeda $P$, Hong $T$, Shore BJ, Kim HK; Study Conducted by the International Perthes Study Group. Feasibility and safety of perfusion MRI for LeggCalvé-Perthes disease. J Pediatr Orthop 2014;34(7):679-82. Crossref

57. Kim HK, Wiesman KD, Kulkarni V, Burgess J, Chen E, Brabham C, Ikram H, Du J, Lu A, Kulkarni AV, Dempsey M, Herring JA. Perfusion MRI in Early Stage of Legg-Calvé-Perthes Disease to Predict Lateral Pillar Involvement: A Preliminary Study. J Bone Joint Surg Am 2014;96(14):1152-60. 